\title{
Parametric invariant Random Matrix Model and the emergence of multifractality
}

\author{
J. A. Méndez-Bermúdez ${ }^{1,2,3}$, Tsampikos $\operatorname{Kottos}^{1,4}$, Doron Cohen ${ }^{2}$ \\ ${ }^{1}$ Max-Planck-Institut für Dynamik und Selbstorganisation, Bunsenstraße 10, D-37073 Göttingen, Germany \\ ${ }^{2}$ Department of Physics, Ben-Gurion University, Beer-Sheva 84105, Israel \\ ${ }^{3}$ Instituto de Física, Universidad Autónoma de Puebla, Apartado Postal J-48, Puebla 72570, México \\ ${ }^{4}$ Department of Physics, Wesleyan University, Middletown, Connecticut 06459-0155, USA
}

\begin{abstract}
We propose a random matrix modeling for the parametric evolution of eigenstates. The model is inspired by a large class of quantized chaotic systems. Its unique feature is having parametric invariance while still possessing the non-perturbative breakdown that has been discussed by Wigner 50 years ago. Of particular interest is the emergence of an additional crossover to multifractality.

PACS numbers: 05.45.Mt, 05.45.Df, 72.15.Rn, 71.30.+h
\end{abstract}

\section{INTRODUCTION}

The analysis of structural changes of eigenstates as a parameter $x$ is varied, has sparked a great deal of research activity for many years [1, 2, 3, 4, 4, 5, 6, 7, 8, 9]. Of particular importance are quantized chaotic or complex systems where the change of $x$ may represent the effect of some externally controlled field (like electric field, gate voltage or magnetic flux). Thus, these studies are relevant for diverse areas of physics ranging from nuclear [1, 10] and atomic physics [1] to quantum chaos [4, 5, 6] and mesoscopics []].

In all these studies, Random Matrix Theory (RMT) played a dominant role as a reference theory that describes the universal properties of the eigenstates of complex systems. RMT was introduced 50 years ago by Wigner as a tool to describe the statistical properties of the eigenvalues of complex nuclei. Until recently, the matrices in the frame of RMT were assumed to be homogeneous, i.e., all matrix elements were set to have identical statistical properties. Under this simplification random matrices are rotationally invariant, a property that simplifies their theoretical analysis. In physical applications this implies that interactions are assumed to be so strong and complex that no other parameters, apart from the symmetry of the Hamiltonian matrix, are relevant. As a result, such random matrices can be associated to the extreme case of maximal chaos, which is known to appear in various physical systems such as heavy nuclei, atoms, metallic clusters, etc. Moreover, one can treat full random matrices as a typical model when describing local statistical properties of spectra and eigenstates in some range of the energy spectrum, typically, in the semiclassical region.

On the other hand, the conventional RMT can not describe important phenomena such as localization of eigenstates, neither can be directly applied to obtain spectra of realistic models. For this reason, much attention has been recently paid to the so-called Wigner Band Random Matrix (WBRM) model which is characterized by the free parameter $b$, that represents the effective bandwidth of a Hamiltonian matrix. Among the important applications of the WBRM model we mention the study of localization in quasi-one-dimensional disordered systems. Also the WBRM model has been applied to the analysis of either chaotic or complex conservative quantum systems that are present in nuclear physics as well as in atomic and molecular physics.

Despite its success, the standard WBRM model has severe limitations in modeling realistic systems. We explain these limitations in section II, and further motivate the introduction of a new RMT ensemble to which we refer as the Winger Lorentzian Random Matrix (WLRM) model. In Sec. III, the WLRM model is shown to have a "parametric invariance" property which is characteristic of any realistic system but is missing in the standard WBRM model. The analysis of the local density of states of the WLRM model is done in Sec. IV. The multifractal properties of the eigenstates of the WLRM ensemble are analyzed in Sec.V. Our conclusions are summarized in Sec. VI.

\section{RMT MODELING}

The pioneering work in this field has been done by Wigner [1], who has motivated the studies of RMT models of the type

$$
\mathcal{H}=\boldsymbol{E}+x \boldsymbol{B} .
$$

Both $\boldsymbol{E}$ and $\boldsymbol{B}$ are real symmetric matrices of size $N \times N$. The elements of the diagonal matrix $\boldsymbol{E}$ are the ordered energies $\left\{E_{n}\right\}$, with mean level spacing $\Delta$, while $\boldsymbol{B}$ is a banded random matrix which is characterized by a band profile $C(r)$. Namely, the entries of $\boldsymbol{B}$ are random numbers that are drawn from a normal distribution with zero mean and variance given by

$$
\left\langle\left|B_{n m}\right|^{2}\right\rangle=C(n-m) .
$$

For the study of spectral statistics of the energy levels it turns out that full matrices $(C(r)=1)$, say of the Gaussian Orthogonal ensemble (GOE), are enough in order to capture the universality which is found in quantized chaotic system. But for the study of parametric evolution of the eigenstates it is essential to take into account 
the band profile, which is dictated by semiclassical considerations. Namely, $C(r)$ is merely the scaled version of a classical power spectrum $\tilde{C}(\omega)$ which is obtained via a Fourier transform of the classical correlation function of the generalized force $\mathcal{F}(t)=-\partial \mathcal{H} / \partial x$.

\section{A. The standard WBRM model}

The standard WBRM model assumes a rectangular band profile:

$$
C(r)=\left\{\begin{array}{ll}
1 & r \leq b \\
0 & r>b
\end{array} .\right.
$$

For this model Wigner has found that the eigenstates undergo a transition from a perturbative Lorentzian-type line shape to a non-perturbative semicircle line-shape. It should be clear that the Wigner Lorentzian can be regarded as the outcome of perturbation theory to infinite order, while the semicircle line-shape is beyond any order of perturbation theory.

The existence of a transition from a perturbative to a non-perturbative line shape is a generic feature of any realistic (quantized) Hamiltonian. In the latter case the semi-circle line-shape is replaced by a semi-classical line shape.

\section{B. The modified WBRM model}

The WBRM model suffers from a serious drawback. Unlike generic canonically quantized Hamiltonians, the statistical properties of its Hamiltonian matrix are not invariant under $x \rightarrow x+$ const. In fact there exists two wise modified versions of the WBRM model [12, 13] which are manifestly $x$ invariant by construction. For example we cite one of them:

$$
\mathcal{H}=\boldsymbol{E}+\cos (x) \boldsymbol{B}_{1}+\sin (x) \boldsymbol{B}_{2}
$$

Here $\boldsymbol{B}_{1}$ and $\boldsymbol{B}_{2}$ are uncorrelated banded matrices. It is quite easy to be convinced that this model is $x$ invariant. One simply has to set $x \rightarrow x+$ const, to expand the $\sin ()$ and the $\cos ()$, to define $\boldsymbol{B}_{1}^{\prime}$ and $\boldsymbol{B}_{2}^{\prime}$, and to observe that they are uncorrelated with the same band profile as $\boldsymbol{B}_{1}$ and $\boldsymbol{B}_{2}$.

However there is a "price" for using such modified model. It is not difficult to prove that the parametric nature of this model is essentially perturbative: The associated local density of states does not exhibit the nonperturbative crossover that has been highlighted in the previous subsection!

\section{The Winger Lorentzian Random Matrix model}

In the present paper we introduce a new RMT ensemble to which we refer as the Winger Lorentzian Random
Matrix (WLRM) model. The Hamiltonian is assumed to have the standard form of Eq.(11), and it is characterized by the band profile

$$
C(r)=\frac{1}{1+(r / b)^{2}} .
$$

There are several good reasons that motivate the introduction and the study of this model, which we are going to clarify:

(1) There is a major class of quantized chaotic systems that can be described using this model.

(2) Unlike the standard WBRM model it has the desired $x$ invariance property that characterizes quantized models.

(3) Unlike the common $x$ invariant version of the WBRM model it exhibits the transition to a nonperturbative line shape.

(4) The emergence of multifractality, which is absent in the WBRM model, is a fascinating issue by itself.

Let us expand on the first point. We recall that the flat band profile of the standard WBRM model is motivated by the realization that many observables (say $\mathcal{F}(t))$ of chaotic systems exhibit "white" power spectrum: $\tilde{C}_{\mathcal{F F}}(\omega) \sim$ const. However, in many cases it is $G(t)=\dot{\mathcal{F}}$ that has the "white" power spectrum [2, 3]. In the latter case the relation $\tilde{C}_{\mathcal{F} \mathcal{F}}(\omega)=\tilde{C}_{G G}(\omega) / \omega^{2}$ implies Lorentzian tails.

\section{PARAMETRIC INVARIANCE}

An important feature of a generic canonically quantized Hamiltonian $\mathcal{H}(Q, P ; x)$ is its parametric $x$ invariance. Given $x^{*}$ we can represent the Hamiltonian by a matrix

$$
\mathcal{H}=\boldsymbol{E}+\delta x \boldsymbol{B}
$$

where $\delta x=x-x^{*}$. If we take two different values of $x^{*}$ we get two different $\boldsymbol{B}$ matrices. But if the two values of $x^{*}$ belong to the same classically small window, then (by definition) the band profile $C(r)$ comes out the same. Still from a quantum mechanical point of view a classically small range of $x$ values is typically regarded as huge. This means that in general, quantum perturbation theory cannot be used in order to describe the parametric evolution within this range.

In Fig. 1 we present $C(r)$ for the standard WBRM model and for the WLRM model. We see that the profile of the perturbation matrix $\boldsymbol{B}$ of the WBRM model is deformed as $x$ is increased, while that of the WLRM model remains the same. We have found out that this $x$ invariance does not hold for other (non-Lorentzian) power law profiles. As examples, in Fig. 2] we present the profiles 


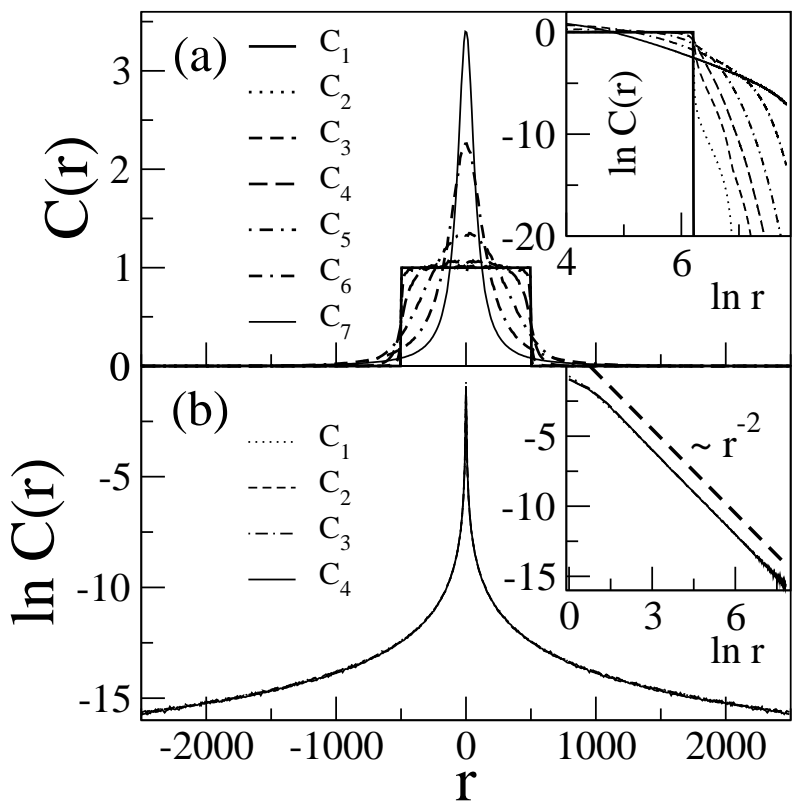

FIG. 1: (a) The band profile $C(r)$ of the standard Wigner model using $b=500$ and $x^{*}=0,0.1,1,3,7,15,31$, and 63 . (b) The band profile $C(r)$ of the Lorentzian Wigner model with $b=1$ and $x^{*}=2.5,27,277$, and 2777. The dashed line in the inset of (b) with decay $\sim r^{-2}$ is plotted to guide the eye.

of $\boldsymbol{B}$ for increasing $x$ in the case of the power-law profile $C(r)=\left[1+(r / b)^{\mu}\right]^{-1}$ with $\mu=0.5$ and 5 . We conclude that the $x$ invariance is a unique property of the WLRM ensemble.

\section{LDOS ANALYSIS}

The local density of states (LDOS) is the major tool for the characterization of the parametric evolution of the eigenstates. The overlap of the eigenstates $|n(x)\rangle$ for a given value of $x$ with the eigenstate $|m(0)\rangle$ of the $x=0$ Hamiltonian is $P(n \mid m)=|\langle n(x) \mid m(0)\rangle|^{2}$. This can be regarded as a distribution with respect to $n$. By averaging over the reference level $m$ we get the line shape $P(n-m)$. Up to trivial scaling this is the LDOS.

The considerations that are required in order to generalize the calculation of the LDOS line shape for a general band profile have been introduced in $[6,8]$. Here, we apply such methodology in order to analyze the parametric evolution of the LDOS for the WLRM model.

For $x=0$ the LDOS is trivially $P(r)=\delta_{r, 0}$ due to orthogonality. As $x$ increases, perturbative tails start to appear. By employing standard first-order perturbation theory we get $P_{\mathrm{FOPT}}(r) \approx 1$ for $r=0$, while

$$
P_{\mathrm{FOPT}}(r)=\frac{x^{2}\left|\boldsymbol{B}_{n m}\right|^{2}}{\left(E_{n}-E_{m}\right)^{2}}=\frac{x^{2}}{\Delta^{2}} \frac{b^{2}}{\left(b^{2}+r^{2}\right)} \frac{1}{r^{2}}
$$

for $r \neq 0$. In the second equality we have substituted

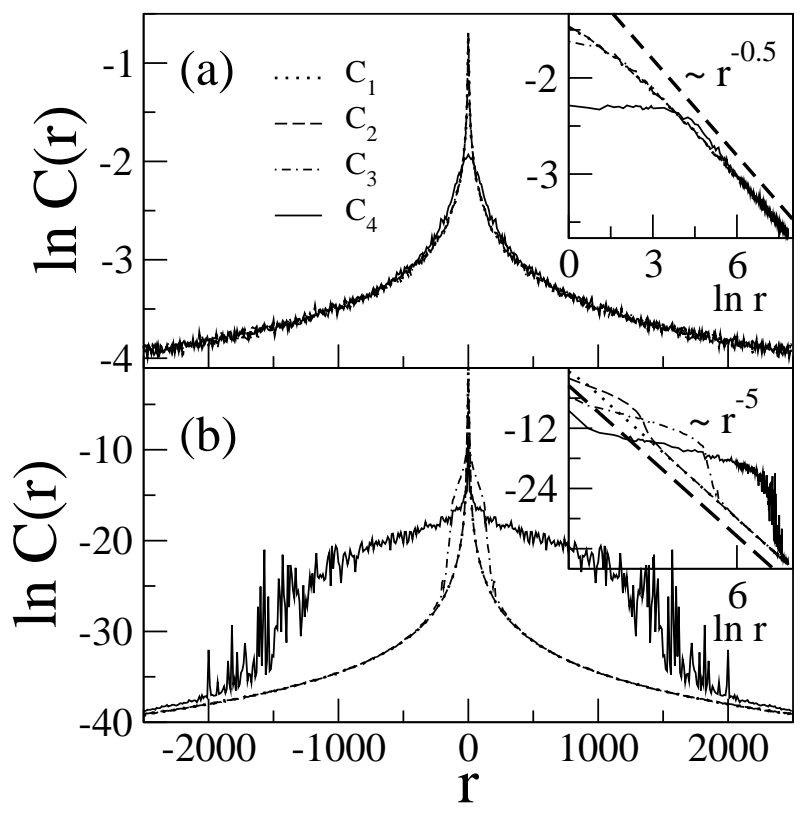

FIG. 2: The band profiles for the power law matrix $C(r)=$ $\left[1+(r / b)^{\mu}\right]^{-1}$ with (a) $\mu=0.5$ and (b) $5 . \quad b=1 . \quad$ In (a) $x^{*}=2.5,27,277$, and 2777. In (b) $x^{*}=0.13,1.4,14$, and 144. The dashed line in the inset of (a) [(b)] with decay $\sim r^{-0.5}\left[\sim r^{-5}\right]$ is plotted to guide the eye.

the matrix elements of $\boldsymbol{B}$ using Eq. (5). The above expression applies for $x<x_{c}$ where $x_{c}$ is the perturbation strength needed to mix neighboring levels only.

For $x>x_{c}$ Wigner had found, in the frame of the WBRM model, that the LDOS line shape can be calculated using perturbation theory to infinite order. In case of the WBRM model one obtains a Lorentzian. Assuming the validity of infinite order perturbation theory we come out with a Lorentzian-type approximation for the LDOS of the WLRM model:

$$
\begin{aligned}
P_{\mathrm{PRT}}(r) & =\frac{x^{2}\left|\boldsymbol{B}_{n m}\right|^{2}}{\Gamma^{2}+\left(E_{n}-E_{m}\right)^{2}} \\
& =\frac{x^{2}}{\Delta^{2}} \frac{b^{2}}{\left(b^{2}+r^{2}\right)} \frac{1}{\left[(\Gamma / \Delta)^{2}+r^{2}\right]} .
\end{aligned}
$$

Eq. (8) is an approximation because all orders of perturbation are treated within a Markovian-like approach (by iterating the first order result) and convergence of the expansion is pre-assumed. The energy scale $\Gamma$ defines the region where a non-perturbative mixing of levels occurs. Inside this region the perturbative profile $P_{\mathrm{PRT}}(r)$ does not describe the actual LDOS lineshape. $\Gamma$ is determined by imposing normalization of $P_{\mathrm{PRT}}(r)$. For the WBRM model it was found [1] that $\Gamma=x^{2} C(1) / \Delta$. For the WLRM model we get

$$
\Gamma=\frac{b \Delta}{2}\left[\sqrt{1+\frac{4 \pi x^{2}}{b \Delta^{2}}}-1\right]
$$




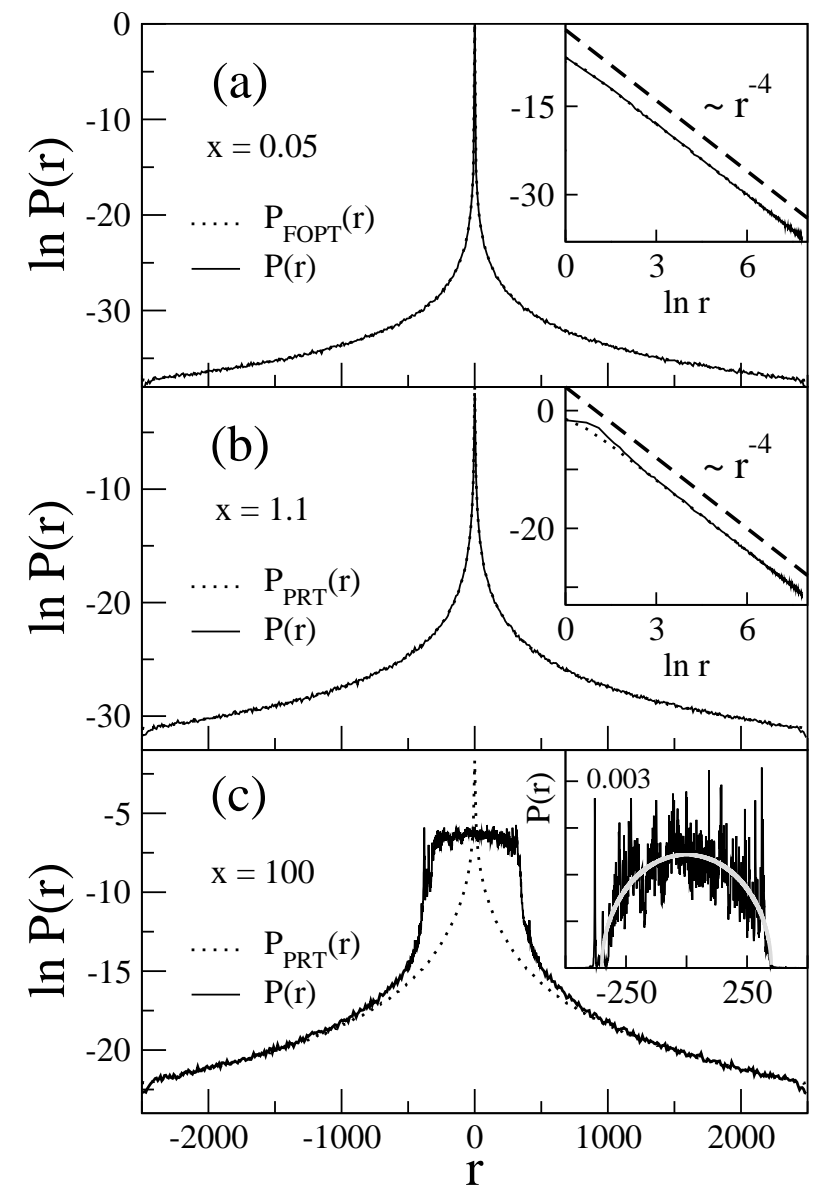

FIG. 3: The LDOS lineshape $P(r)$ in the (a) standard perturbative, (b) extended perturbative, and (c) non-perturbative regimes for the WLRM model with $b=1, \Delta=1$, and $N=5000$. For this set of parameters $x_{c} \approx 0.8$ and $x_{\mathrm{prt}} \approx 1.5$. The first-order perturbation theory profile $P_{\mathrm{FOPT}}(r)$ from Eq. (7) is included in (a). The Wigner-type lineshape $P_{\mathrm{PRT}}(r)$ from Eq. [8], which is expected to be valid in the perturbative regime, is also included (no fitting parameters) in (b) and (c). The dashed line in the insets of (a) and (b) with decay $\sim r^{-4}$ is plotted to guide the eye. The gray line in the inset of (c) is a semicircle fitting to $P(r)$.

Obviously, for $\Gamma \ll \Delta$ the (infinite-order) LDOS profile $P_{\mathrm{PRT}}(r)$ reduces to the standard first-order perturbation theory expression $P_{\mathrm{FOPT}}(r)$. Therefore $x_{c}$ can be determined by the condition $\Gamma\left(x_{c}\right) \approx \Delta$, leading to

$$
x_{c} \approx \frac{\Delta}{\sqrt{\pi}} \sqrt{1+\frac{1}{b}} .
$$

In Fig. 3] we display our numerical results for $P(r)$ for the WLRM model with $b=1, \Delta=1, N=5000$, and various perturbation strengths $x$. We see that the agreement with the perturbative expression (8) persist up to some perturbation strength $x_{\mathrm{prt}}$. Above $x_{\mathrm{prt}}$ the LDOS lineshape $P(r)$ becomes semicircle in complete analogy with the WBRM model scenario.
We want to find the value $x_{\text {prt }}$ up to which the perturbative expression $P_{\mathrm{PRT}}(r)$ describes reasonably good the LDOS lineshape. To this end we compare the dispersion of $P_{\mathrm{PRT}}(r)$,

$$
\delta E_{\mathrm{PRT}}=\Delta \times \sqrt{\sum_{r} r^{2} P_{\mathrm{PRT}}(r)},
$$

to the dispersion of the actual LDOS [6]

$$
\delta E=x \sum_{r \neq 0} C(r) .
$$

Expressions for $\delta E_{\mathrm{PRT}}$ and $\delta E$ in case of our WLRM model can be obtained by replacing the sums above by integrals:

$$
\begin{gathered}
\delta E_{\mathrm{PRT}} \approx x \sqrt{\pi} b(b+\Gamma / \Delta)^{-1 / 2}, \\
\delta E \approx x \sqrt{2 b}[\pi / 2-\arctan (1 / b)]^{1 / 2} .
\end{gathered}
$$

Note that for small $x, \delta E_{\mathrm{PRT}}=\delta E \approx x(\pi b)^{1 / 2} . \delta E$ is a linear function of $x$ for all perturbation strengths while for large enough $x \delta E_{\mathrm{PRT}}$ becomes sublinear: $\delta E_{\mathrm{PRT}} \propto$ $x^{1 / 2}$. See Fig. 4 The border $x_{\text {prt }}$ is identified as the perturbation strength for which $\delta E_{\mathrm{PRT}}\left(x_{\mathrm{prt}}\right) \approx \gamma \delta E\left(x_{\mathrm{prt}}\right)$, where $\gamma<1$ :

$$
x_{\mathrm{prt}} \approx \Delta \sqrt{b} \frac{\sqrt{\pi-2 \gamma^{2}[\pi / 2-\arctan (1 / b)]}}{2 \gamma^{2}[\pi / 2-\arctan (1 / b)]} .
$$

We typically use $\gamma=0.8$.

The validity of Eqs. (8), (13), and (14) is confirmed in Fig. 4 for $b=100, \Delta=1$, and $N=5000$. There, we observe excellent agreement between the expressions for $\delta E_{\mathrm{PRT}}$ and $\delta E$ and the corresponding numerics. To verify the validity of Eq. (8) we compare the $50 \%$ probability width (defined as the energy width of the central $r$ region that contains $50 \%$ of the probability) of $P(r)$ to that of $P_{\mathrm{PRT}}(r)$ finding reasonable good agreement for $x<x_{\mathrm{prt}}$, as expected.

Does the LDOS analysis capture all the features of the eigenstates? The answer turns out to be negative. In case of the WBRM model there is still another regime which is not captured by the LDOS analysis. Namely, for $x>x_{\infty}$ where $x_{\infty}=b^{3 / 2} x_{c}$ the eigenstates of the Hamiltonian become exponentially localized. This is the well known Anderson (strong) localization effect. For $x>x_{\infty}$ the Hamiltonian is essentially $\mathcal{H}=\boldsymbol{B}$ as if the diagonal $\boldsymbol{E}$ does not exist. Do we have an analogous type of crossover in case of the WLRM model? The answer must be positive because we know 14 that in case of the WLRM model, the Hamiltonian $\mathcal{H}=\boldsymbol{B}$ has multifractal (rather than localized) eigenstates.

\section{MULTIFRACTALITY}

The multifractal structure of the eigenstates is commonly characterized by the fractal dimension $D_{2}$ which 


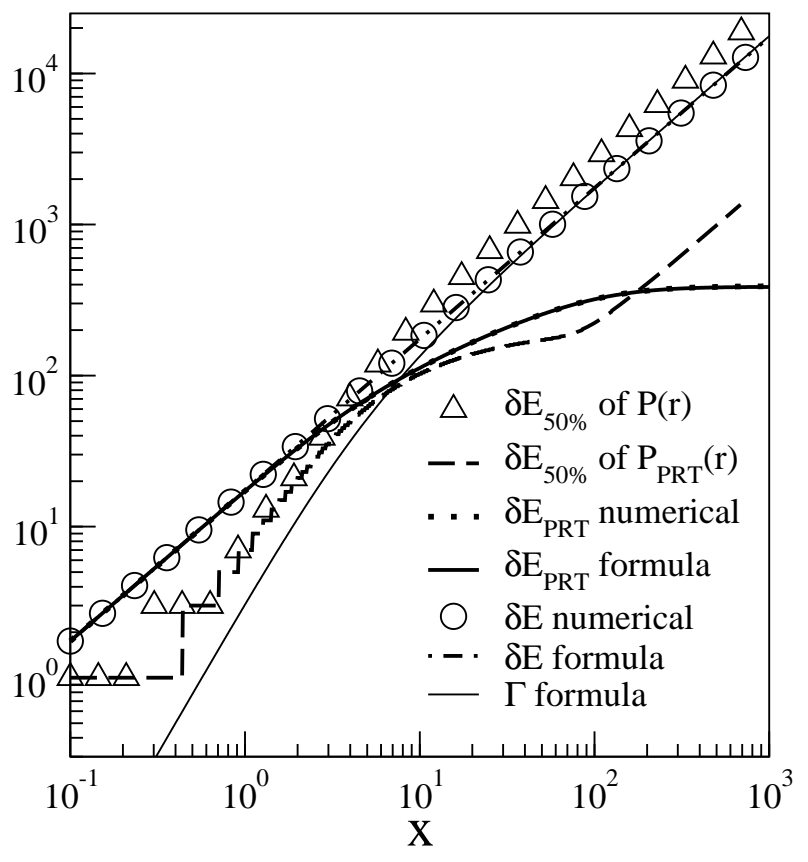

FIG. 4: $\delta E_{\mathrm{PRT}}$ and $\delta E$ as a function of $x$ for the WLRM model with $b=100, \Delta=1$, and $N=5000$. The $50 \%$ probability width of $P(r)(\triangle)$ and of $P_{\mathrm{PRT}}(r)$ (dashed line) are plotted to verify the validity of Eq. (8) for $x<x_{\mathrm{prt}}$. $\delta E_{\mathrm{PRT}}$ (dotted line) and $\delta E(\circ)$ were obtained from $\delta E_{\mathrm{PRT}}^{2}=\Delta \sum_{r} r^{2} P_{\mathrm{PRT}}(r)$ and $\delta E^{2}=\Delta \sum_{r} r^{2} P(r)$, respectively. The numerically obtained $\delta E_{\mathrm{PRT}}$ (dotted line) is hardly visible in the plot because it lies on top of $\delta E_{\mathrm{PRT}}$ from Eq. (13). We also show $\Gamma$ (thin full line) from Eq. (9). For this set of parameters $x_{c} \approx 0.57$ and $x_{\mathrm{prt}} \approx 5.35$.

is associated with the scaling of the inverse participation ratio. Given an eigenstate of $\mathcal{H}$ which is represented by a column vector $\Psi_{n}$ we define the participation ratio as

$$
\mathcal{N}_{2}=\left[\sum_{n}\left|\Psi_{n}\right|^{4}\right]^{-1} .
$$

The exponent $D_{2}$ is defined via the scaling relation

$$
\overline{\mathcal{N}_{2}} \propto N^{D_{2}} .
$$

where $\overline{\mathcal{N}_{2}} \equiv \exp \left(\left\langle\ln \mathcal{N}_{2}\right\rangle\right)$. The measure $\overline{\mathcal{N}_{2}}$ constitutes an estimate for the typical number of non-zero eigenfunction components of the column vector. For a localized state it equals a constant number and hence $D_{2}=0$, while for an extended non-fractal state it is proportional to the size of the matrix $N$ and hence $D_{2}=1$. In general one finds $0<D_{2}<1$. The fractal dimension $D_{2}$ manifests itself in a variety of physical circumstances. As examples we mention the conductance distribution in metals [15, 16], the statistical properties of the spectrum [16], the anomalous spreading of a wave-packet, the spatial dispersion of the diffusion coefficient [17] and the anomalous scaling of delay times [18].

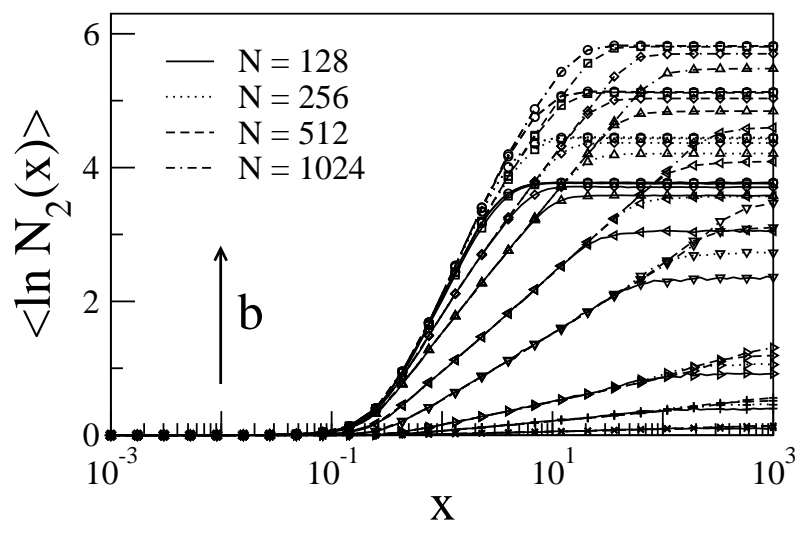

FIG. 5: $\left\langle\ln \mathcal{N}_{2}(x)\right\rangle$ as a function of $x$ for $N=128,256,512$, and 1024. The bandwidth is $b=100(\circ), 40(\square), 10(\diamond), 4(\triangle)$, $1(\triangleleft), 0.4(\nabla), 0.1(\triangleright), 0.04(+)$, and $0.01(\times)$.

In 14] it was shown that the fluctuations of $\mathcal{N}_{2} / \overline{\mathcal{N}_{2}}$ for $\mathcal{H}=\boldsymbol{B}$ are characterized by a universal probability distribution. A theoretical estimation [19] gives

$$
D_{2}=\left\{\begin{array}{cc}
1-(\pi b)^{-1} & b \gg 1 \\
2 b & b \ll 1
\end{array} .\right.
$$

We notice that $D_{2}=D(b)$ gives a global fit for the fractal dimension, where we define

$$
D(b)=\frac{1}{1+(2.34 b)^{-1}} .
$$

Note that (19) is also in agreement with the numerical found [20] value $D_{2} \approx 0.7$ for $b=1$. For sake of later analysis we have found that the associated proportionality factor in Eq. (17) is $\exp (-G(b))$ where

$$
G(b) \approx \frac{1}{1+(1.23 b)^{-1}} .
$$

Thus, based on the above, we may say that for $x=\infty$ we expect to have multifractal eigenstates. We turn now to discuss the more general case of finite $x$. We want to see how the multifractality emerges as we increase $x$ form zero to infinity.

In the numerics we assume without loss of generality that the mean level spacing is $\Delta=1$. This implies that for $b>1$ the threshold for mixing of levels is $x_{c}=\pi^{-1 / 2}$, while for $b<1$ it is $x_{c}=(\pi b)^{-1 / 2}$. Our main interest is in the non-trivial regime $x>x_{c}$. In Fig. 5 we plot $\ln \overline{\mathcal{N}_{2}}(x)$ for several values of $b$ and $N$. We calculate the average using $25 \%$ of the eigenstates at the middle of the spectra from a number of realizations of $\boldsymbol{B}$ summing up a total of 100, 000 data values for each $(b, N)$.

Looking at Fig. [5 we see that for $x=0$ we have $\ln \overline{\mathcal{N}_{2}}=0$ because all the eigenstates have only one component. We observe that this zero value persists up to a point $x=x_{0}(b)$. In principle one can argue that $x_{0}(b)$ should be of the order $x_{c}$ and (for $b>1$ ) not larger than 


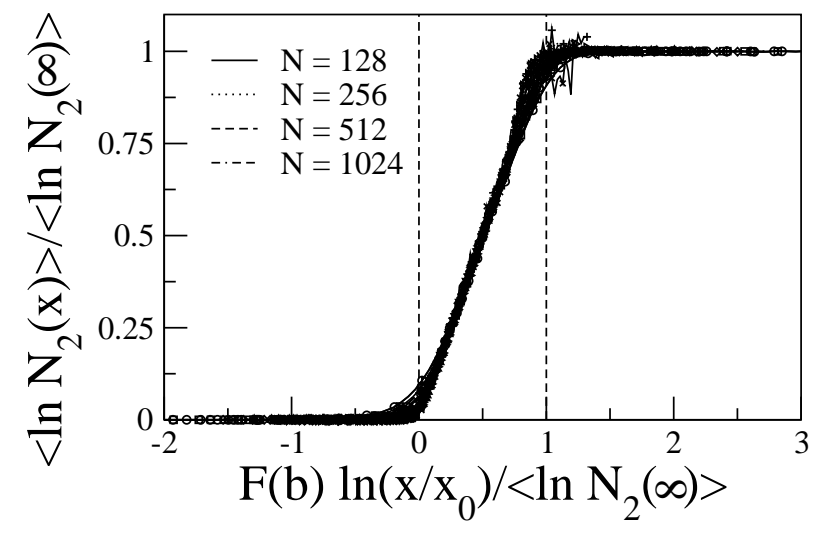

FIG. 6: The scaled version of Fig. 5$]\left\langle\ln \mathcal{N}_{2}(x)\right\rangle /\left\langle\ln \mathcal{N}_{2}(\infty)\right\rangle$ is plotted against $F(b) \ln \left(x / x_{0}\right) /\left\langle\ln \mathcal{N}_{2}(\infty)\right\rangle$. The parametric crossovers at $x=x_{0}$ and $x=x_{\infty}$, are indicated by dashed lines.

$x_{\mathrm{prt}} \approx \sqrt{b} x_{c}$. However, from Eq. (19) it is clear that for $b>100$ we already have $D_{2} \approx D(\infty)=1$. Therefore the $b$ dependence of $x_{0}$ can be neglected, and in practice cannot be detected. We shall see below that for any practical purpose one can take $x_{0} \approx 0.15$.

As $x$ is increased beyond $x_{0}$ the participation ratio $\mathcal{N}_{2}$ becomes larger. As long as $x$ is not too large the $\boldsymbol{E}$ term in the Hamiltonian dominates, and therefore the size of the matrix is of no importance. Indeed, we see that the curves in Fig. [5 are $N$ independent for small $x$ values. From this plot we find that the slope of the curves is given by

$$
F(b) \approx\left\{\begin{array}{cc}
0 & b<0.1 \\
0.57+0.2 \ln (b) & b \geq 0.1
\end{array} .\right.
$$

For large enough $x$, the value of $\ln \overline{\mathcal{N}_{2}}(x)$ saturates to the $x=\infty$ multifractal result. We call the crossover point $x_{\infty}$. Using the knowledge of both the $x<x_{\infty}$ behavior (as described in the previous paragraph), and the $x>x_{\infty}$ behavior (saturation), we deduce that

$$
x_{\infty}(N ; b)=x_{0} \mathrm{e}^{-G(b) / F(b)} N^{D(b) / F(b)} .
$$

Putting together all the above findings we end up with the following global scaling relation for the participation ratio:

$$
\overline{\mathcal{N}_{2}}(x)= \begin{cases}\approx 1 & x<x_{0} \\ \left(x / x_{0}\right)^{F(b)} & x_{0}<x<x_{\infty}(N ; b) \\ \mathrm{e}^{-G(b)} N^{D(b)} & x>x_{\infty}(N ; b)\end{cases}
$$

In Fig. 66 we demonstrate this scaling. We plot $\ln \overline{\mathcal{N}}_{2}(x) / \ln \overline{\mathcal{N}}_{2}(\infty)$ as a function of the scaled variable $F(b) \ln \left(x / x_{0}\right) / \ln \overline{\mathcal{N}}_{2}(\infty)$. We see clearly the trivial crossover at $x=x_{0}$ and the non-trivial crossover to multifractality at $x=x_{\infty}$.

Possibly it is more instructive to describe the behavior of $\overline{\mathcal{N}_{2}}$ as a function of $N$ for a given $x$. The interesting case is to have a fixed value of $x$ which is much larger than $x_{0}$. As we increase $N$ we have a multifractal growth $\overline{\mathcal{N}_{2}} \propto N^{D_{2}}$. This goes on as long as $x_{\infty}(N ; b)$ remains smaller than $x$. After that $\overline{\mathcal{N}_{2}}$ reaches saturation, as implied by Eq. (23). The saturation value is related to the "width" of the LDOS, and hence has an algebraic dependence on the dimensional strength $\left(x / x_{0}\right)$ of the perturbation [8]. It is pleasing to note that for $b \sim 1$ we observe $F(b) \approx 2 / 3$ which is related to considerations as in [8].

\section{CONCLUSIONS}

We have analyzed the parametric evolution of eigenstates for the WLRM model. Both, the standard WBRM and the WLRM models, exhibit a crossover from a perturbative regime where the LDOS is Lorentzian-like to a non-perturbative regime where the LDOS is semicirclelike. However, there is in both cases an additional crossover which is not captured by the conventional LDOS analysis: In the case of the standard WBRM model it is the well studied crossover to an Anderson localization regime, where the eigenstates become exponentially localized; In the case of the WLRM model it is the emergence of multifractality.

We have also shown that the WLRM model possess the $x$ invariance property, absent in the standard WBRM model and in other models that do not have a Lorenzian band profile. Both the non-perturbative crossover and the $x$ invariance property characterize realistic quantized Hamiltonians.

Acknowledgments. This research was supported by a grant from the GIF, the German-Israeli Foundation for Scientific Research and Development, and by the Israel Science Foundation (grant No.11/02).
[1] E. Wigner, Ann. Math. 62, 548 (1955); 65, 203 (1957).

[2] A. Barnett, D. Cohen and E.J. Heller, Phys. Rev. Lett. 85, 1412 (2000); J. Phys. A 34, 413 (2001).

[3] J. A. Méndez-Bermúdez, T. Kottos, and D. Cohen, Phys. Rev. E 72, 027201 (2005).
[4] J. A. Méndez-Bermúdez, G. A. Luna-Acosta, and F. M. Izrailev, Physica E 22, 881 (2004); Phys. Rev. E 68, 066201 (2003).

[5] L. Benet, et. al., J. Phys. A: Math. Gen. 36, 1289 (2003); L. Benet, et. al., Phys. Lett. A 277, 87 (2000); F. Bor- 
gonovi, I. Guarneri, F. M. Izrailev, Phys. Rev. E 57, 5291 (1998).

[6] D. Cohen and T. Kottos, Phys. Rev. E 63, 036203 (2001).

[7] R. O. Vallejos, C. H. Lewenkopf, and Y. Gefen, Phys. Rev. B 65, 085309 (2002); G. Murthy, et. al., ibid, 69, 075321 (2004); L. G. G. V. Dias da Silva, et. al., ibid, 69, 075311 (2004).

[8] D. Cohen and E. J. Heller, Phys. Rev. Lett. 84, 2841 (2000).

[9] M. Hiller, T. Kottos, and T. Geisel, submitted (2006).

[10] V. K. B. Kota, Phys. Rep. 347, 223 (2001); V. Zelevinsky, et. al., Phys. Rep. 276, 85 (1996).

[11] L. Kaplan and T. Papenbrock, Phys. Rev. Lett. 84, 4553 (2000); V. V. Flambaum, A. A. Gribakina, G. F. Gribakin, M. G. Kozlov, Phys. Rev. A 50, 267 (1994).

[12] E.J. Austin and M. Wilkinson, Nonlinearity 5, 1137 (1992).

[13] H. Attias and Y. Alhassid, Phys. Rev. E 52, 4776 (1995).

[14] F. Evers and A. D. Mirlin, Phys. Rev. Lett. 84, 3690
(2000); E. Cuevas, M. Ortuno, V. Gasparian, and A. Perez-Garrido, Phys. Rev. Lett. 88, 016401 (2002).

[15] B. L. Altshuler, V. E. Kravtsov, I. V. Lerner, in Mesoscopic Phenomena in Solids, ed. B. L. Altshuler, P. A. Lee, R. A. Webb (North Holland, Amsterdam, 1991).

[16] D. Braun, E. Hofstetter, G. Montambaux, and A. MacKinnon, Phys. Rev. Lett. 64, 155107 (2001); K. Slevin and T. Ohtsuki, ibid. 82, 382 (1999); K. A. Muttalib and P. Wölfle, ibid. 83, 3013 (1999); P. Markos, ibid. 83, 588 (1999); B. Shapiro, ibid. 65, 1510 (1990).

[17] J. T. Chalker and G. J. Daniell, Phys. Rev. Lett. 61, 593 (1988); B. Huckestein and R. Klesse, Phys. Rev. B 59, 9714 (1999).

[18] J. A. Méndez-Bermúdez and T. Kottos, Phys. Rev. B 72, 064108 (2005).

[19] A. D. Mirlin, Y. V. Fyodorov, F.-M. Dittes, J. Quezada, and T. H. Seligman, Phys. Rev. E 54, 3221 (1996).

[20] I. Varga, Phys. Rev. E 66, 094201 (2002). 